\title{
Platelet-rich plasma induces increased expression of G1 cell cycle regulators, type I collagen, and matrix metalloproteinase-1 in human skin fibroblasts
}

\author{
JAE-WE CHO* ${ }^{*}$ SUNG-AE KIM* and KYU-SUK LEE \\ Department of Dermatology, Keimyung University School of Medicine, Daegu 700-712, Republic of Korea
}

Received July 24, 2011; Accepted September 9, 2011

DOI: $10.3892 /$ ijmm.2011.803

\begin{abstract}
Platelet-rich plasma (PRP) is derived from fresh whole blood, which contains a high concentration of platelets. Recently, PRP has been used for skin wound healing and rejuvenation. However, the molecular mechanisms underlying PRP-inducing wound healing processes are still largely unknown. The aim of this study is to evaluate the effect of PRP on the expression of G1 cell cycle regulatory proteins, type I collagen, matrix metalloproteinase-1 (MMP-1), and MMP-2 in human skin fibroblasts (HSF). We performed a cell proliferation and a migration assay, immunoblotting, and a chloramphenicol acetyltransferase (CAT) assay in PRP-treated human skin fibroblasts. PRP treatment induced increased rates of cell proliferation and cell migration. Expression of cyclin A protein was increased by a low concentration $(0.5 \%)$ of PRP-treated HSF. In addition, expression of Rb, cyclin E, and cyclin-dependent kinase 4 proteins was increased by a high concentration (5\%) of PRP-treated HSF. High concentration of PRP induced an up-regulation of type I collagen, MMP-1, and MMP-2 expression in HSF. Taken together, PRP treatment induced an increase in expression of G1 cell cycle regulators, type I collagen and MMP-1, thereby accelerating the wound healing process.
\end{abstract}

\section{Introduction}

Platelet-rich plasma (PRP) is a blood-derived fraction containing at least 100 thousand platelets (1). In clinical applications PRP has been used to facilitate bone proliferation in nonunion fractures of joint replacement of orthopedic surgery, regeneration of periodontal ligaments, and to accelerate the wound healing process in diabetic ulcers (2-4). However, the

Correspondence to: Dr Kyu-Suk Lee, Department of Dermatology, Keimyung University School of Medicine, 194 DongSan-Dong Jung-Gu, Daegu 700-712, Republic of Korea

E-mail: janylove99@hanmail.net

*Contributed equally

Key words: platelet-rich plasma, type I collagen, matrix metalloproteinase, fibroblasts precise molecular mechanisms of the PRP-induced wound healing process remain to be elucidated.

Wound healing processes are composed of coagulation, inflammation, migration/proliferation, and remodeling phases (5). The migration/proliferation phase and remodeling phase are the key steps in proper wound healing $(6,7)$. Epithelial resurfacing, fibroplasias, angiogenesis, and extracellular matrix (ECM) deposition occur synchronously during migration/proliferation and remodeling phases. Deposition of ECM proteins is regulated by synthesis and degradation of type I collagen. Degradation of type I collagen is mainly mediated by matrix metalloproteinase-1 (MMP-1) and MMP-2 $(8,9)$. In contrast, chronic wounds, such as those of diabetic foot ulcer and venous leg ulcer, show impairment of the wound healing process. Impairment of keratinocyte and fibroblast migration, lack of angiogenesis, and excessive deposition of ECM proteins are observed in chronic wound areas (10). Thus, PRP application on a chronic wound will promote wound healing processes by growth factors.

A defect in fibroblast cell-cycling activity in chronic pressure ulcers has been described $(10,11)$. Fibroblasts within the chronic wounds show features of arrested cell cycle. For achievement of wound healing processes, it is important to induce activation of the arrested cell cycles of fibroblasts within chronic wounds. Proliferation of fibroblasts is precisely regulated by cell cycle regulatory proteins $(12,13)$. Cell cycle regulatory proteins are composed of two protein classes, the cyclins and their kinase partners, Cdks. Two families of cyclins are successively activated during the G1 phase, and, thus, also named G1 cyclins. G1 cyclins are composed of D-type and E-type cyclins. D-type cyclins assemble with their catalytic partners, Cdk4 and Cdk6, followed by cyclin $\mathrm{E}$ interaction with its catalytic partner, Cdk2. The subsequent cyclin-Cdk complexes drive cell cycles from the $\mathrm{G} 1$ phase into the $\mathrm{S}$ phase by phosphorylation of $\mathrm{Rb}$, resulting in cell proliferation. Indeed, cyclin D1-Cdk4/6 and cyclin E-Cdk2 are the key regulatory proteins in the progression of G1/S transition phases. Actually, arrest of the cell cycle and inactivation of fibroblasts showing decreasing expression of cyclin D1 are commonly observed within chronic wound areas, and result in wound chronicity. Therefore, for acceleration of wound healing, evaluation of the effect of PRP on the expression of G1 cell cycle regulatory proteins in human skin fibroblasts (HSF) is important. 
In this study, we investigated the effect of PRP on cell migration, proliferation, expression of G1 cell cycle regulatory proteins, and expression of type I collagen, MMP-1, and MMP-2 in fibroblasts.

\section{Material and methods}

Materials. Antibodies against type I collagen and MMP-1 were purchased from Abcam (Cambridge, UK), MMP-2 from Chemicon (Temecula, CA), and $\beta$-actin and cell cycle regulatory proteins $(\mathrm{Cdk} 2, \mathrm{Cdk} 4$, cyclin A, cyclin D1, cyclin E) were obtained from Santa Cruz Biotechnology (Santa Cruz, $\mathrm{CA}$ ). Anti-Rb antibody was purchased from Pharmingen (BD Biosciences, CA). Antibodies against phospho-ERK (p-ERK), ERK, phospho-p38 (p-p38), p38, phospho-JNK (p-JNK), and JNK were purchased from Cell Signaling (Beverly, MA). SB203580 and PD98059 were purchased from Biomol (Plymouth Meeting, PA) and Calbiochem (La Jolla, CA), respectively.

Cell cultures. Human skin fibroblasts were maintained at $37^{\circ} \mathrm{C}$ in a humidified atmosphere of $95 \%$ air and $5 \% \mathrm{CO}_{2}$ in Eagle's minimum essential medium supplemented with $10 \%$ heatinactivated fetal bovine serum, $2 \mathrm{mM}$ glutamine, $100 \mathrm{U} / \mathrm{ml}$ penicillin and $100 \mu \mathrm{g} / \mathrm{ml}$ streptomycin. For experiments, cells $\left(5 \times 10^{4}\right.$ cells $\left./ \mathrm{ml}\right)$ were seeded in culture dishes, and maintained in the tissue culture incubator.

Preparation of activated PRP. PRP was prepared using a PRP kit (MyCells ${ }^{\circledast}$ autologous preparation kit; Israel). Briefly, blood was collected into tubes containing acid-citrate-dextrose solution formula A (1:4, vol:vol) anticoagulant, from healthy adult volunteers $(n=2)$ after receiving informed consent. The citrated blood was centrifuged in a standard laboratory centrifuge for $10 \mathrm{~min}$ at $3,000 \mathrm{rpm}$. The plasma supernatant was used as platelet-poor plasma, and the thrombocyte pellet in $7.0 \mathrm{ml}$ of plasma was used as platelet-rich plasma. Before treatment, a 10:1 (vol/vol) mixture of platelet-rich plasma and $\mathrm{CaCl}_{2}$ was incubated at room temperature for $5 \mathrm{~min}$. All tools of blood sampling and centrifugation were sterilized by autoclaving.

Cell proliferation assay. Human skin fibroblasts were seeded at a density of $15 \times 10^{4}$ cells/well in 6 -well culture plates. The cells were cultured using serum-free DMEM supplemented with 0 (control), $0.05,0.5,1$ or $2 \%$ activated PRP for 3 days. The cultured cells were assayed for proliferation using the trypan blue exclusion method. Briefly, cells were washed with phosphate-buffered saline and stained with trypan blue dye. Viable cells were observed as light reflected cells, but dead cells were observed as dark cells. The viable cell numbers were measured under an inverted phase contrast microscope.

Migration assay. For the measurement of cell migration, confluent fibroblasts, which were kept in serum-free medium for $24 \mathrm{~h}$, were wounded with a plastic micropipette tip having a large orifice. After washing, the medium was replaced by serum-free medium or serum-free media supplemented with $0.05,0.5,1$ or $2 \%$ activated PRP for $24 \mathrm{~h}$. Photographs of the wounded areas were taken every $24 \mathrm{~h}$ by phase-contrast microscopy under crystal violet staining.
Western blot analysis. Whole cell extracts were prepared in lysis buffer [10 mM Tris (pH 7.4), $5 \mathrm{mM}$ EDTA, $130 \mathrm{mM} \mathrm{NaCl}, 1 \%$ Triton X-100, phenylmethylsulphonyl fluoride (PMSF, $10 \mu \mathrm{g} / \mathrm{ml}$ ), aprotinin $(10 \mu \mathrm{g} / \mathrm{ml})$, leupeptin $(10 \mu \mathrm{g} / \mathrm{ml}), 5 \mathrm{mM}$ phenanthroline and $28 \mathrm{mM}$ benzamidine-HCl]. The protein concentration of extracts was estimated with the Bradford reagent (Bio-Rad, Hercules, CA, USA) using bovine serum albumin as the standard. Equal amounts of protein ( $40 \mu \mathrm{g} / \mathrm{lane})$ were resolved by 6.5-12\% sodium dodecyl sulfate-polyacrylamide gel electrophoresis, and transferred onto a nitrocellulose membrane. The membrane was then washed with Tris-buffered saline $(10 \mathrm{mM}$ Tris, $150 \mathrm{mM} \mathrm{NaCl}$ ) containing $0.05 \%$ Tween-20 (TBST) and blocked in TBST containing 5\% non-fat dried milk. The membrane was further incubated with respective specific antibodies. The membrane was continuously incubated with appropriate secondary antibodies coupled to horseradish peroxidase, and developed using the ECL Western detection reagents kit (Amersham Pharmacia Biotech, Piscataway, NJ, USA).

Transfection and chloramphenicol acetyltransferase (CAT) assay. The construct containing the $3.5-\mathrm{kb}$ COL1A2 promoter fused to the CAT gene (pMS-3.5/CAT) was derived from a 3.5-kb EcoRI/SphI genomic subclone that spans the region from position -3500 to +58 of the COL1A2 promoter. After conversion of the EcoRI site to BamHI, the eukaryotic insert was excised with BamHI and HindIII, and was subcloned into a similarly digested polylinker of the expression vector p8-CAT-a derivative of the pEMBL plasmid. Thereafter, deletion mutants were generated in the parental pUC18 subclones and subsequently transferred to the expression vector. The transfections were carried out using the calcium phosphate/ DNA co-precipitation method followed by a $1 \mathrm{~min}(15 \%)$ glycerol shock. Four hours after transfection, cells were treated with PRP. After $24 \mathrm{~h}$ of incubation, cells were harvested, as previously described. CAT activity was determined by incubating cell extracts with $\left[{ }^{14} \mathrm{C}\right]$ chloramphenicol, followed by separation of the acetylated and non-acetylated forms by thin-layer chromatography. CAT activity in the cell extracts was calculated as the radioactivity in the acetylated forms expressed as a percentage of total radioactivity in each sample.

\section{Results}

Effect of PRP on cell proliferation rates of human skin fibroblasts. Increased proliferation of skin fibroblasts is an important factor for wound healing. It is unclear whether PRP treatment induces high rates of proliferation of skin fibroblasts, compared with non-treated fibroblasts. We analyzed the effect of PRP on the proliferation rates of HSF. PRP treatment resulted in a dosedependent increase of the proliferation rates of skin fibroblasts (Fig. 1). In addition, migration of fibroblasts plays important roles in promotion of wound healing. In order to examine whether PRP treatment exhibits more effectiveness on migration of fibroblasts compared with non-treated PRP, confluent fibroblasts cells were scratched using a plastic micropipette and were then cultured for 1 day under various concentrations of PRP. PRP treatment resulted in markedly increased rates of migration of fibroblasts, in a concentration-dependent manner (Fig. 2). 


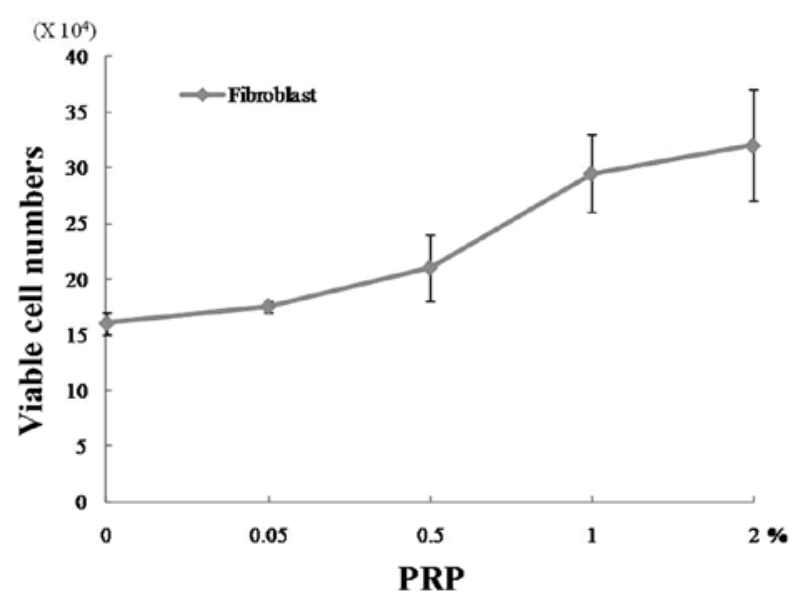

Figure 1. Increased cell proliferation rates of PRP-treated fibroblasts. Cells were plated at a density of $40 \times 10^{4} / \mathrm{cm}^{2}$ in 6 -well plates. Cells were cultured with DMEM without serum for 2 days. Medium with various concentrations of PRP were added and cells were cultured for 3 days. Viable cells were counted using trypan blue exclusion assay.

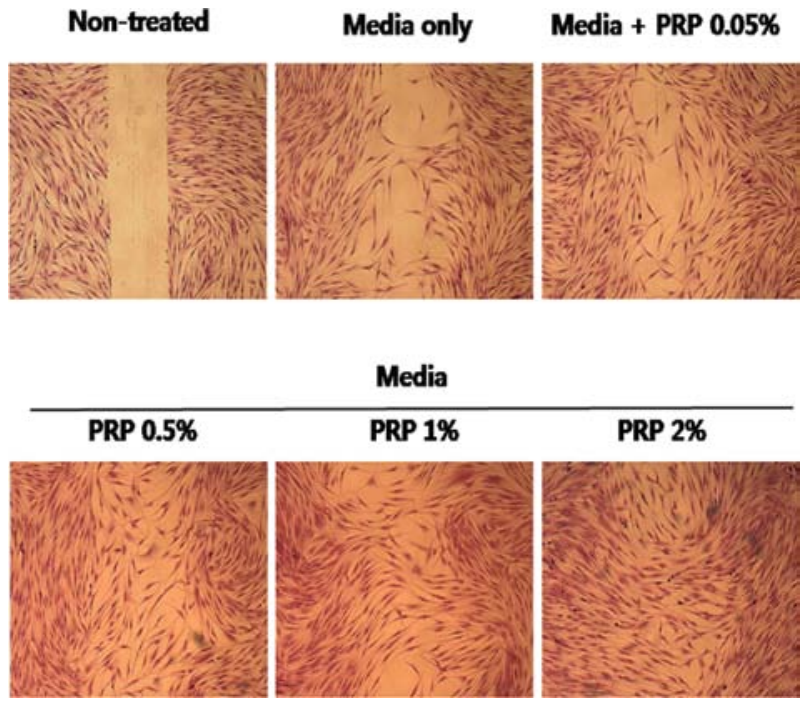

Figure 2. Increased cell migration activities of PRP-treated fibroblasts. Confluent fibroblasts were scratched with a plastic micropipette and cells were cultured with DMEM medium or DMEM medium with various concentrations PRP for 1 day and migration of cells was observed using an inverted microscope.

Expression of cell cycle regulatory proteins in $H S F$ by $P R P$ treatment. Cell cycle regulatory proteins play important roles in proliferation and migration in HSF. We assessed whether PRP treatment promotes proliferation and migration activities of fibroblasts through up-regulation of G1/S transition regulatory proteins. For determination of basal expression levels of cell cycle regulatory proteins in fibroblasts, cells were cultured without fetal bovine serum (FBS) for 3 days. After serum starvation for 3 days, cells were cultured under 5\% FBS, $0.05 \%$ PRP or $0.5 \%$ PRP with DMEM (Fig. 3). Expression levels of G1/S transition regulatory proteins, such as cyclin D1, cyclin E, Cdk2, and Cdk4, were decreased in serum-starved skin fibroblasts. Low concentrations $(0.05-0.5 \%)$ of PRP induced increased expression of cyclin A in fibroblasts, but not cyclin D1, cyclin E, Cdk2, and Cdk4. Of particular interest, a

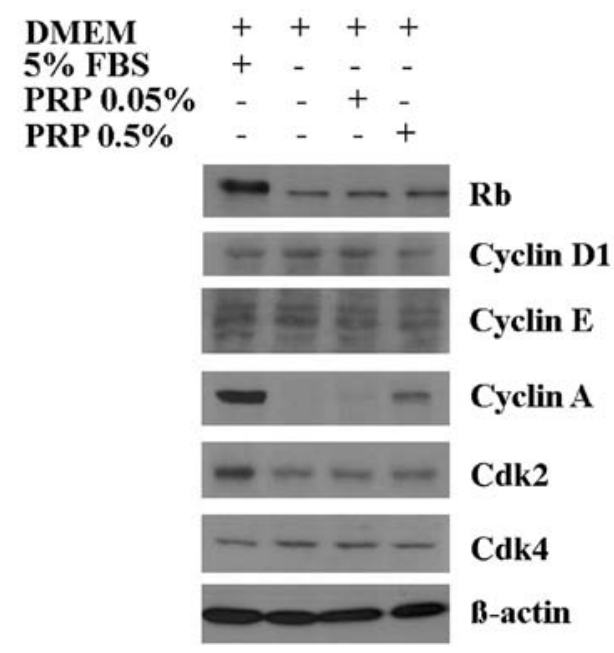

Figure 3. Expression of cell cycle regulatory proteins in PRP (low concentrations)-treated fibroblasts. Cells were cultured without serum for 3 days, followed by addition of $5 \%$ FBS, $0.05 \%$ PRP or $0.5 \%$ PRP to culture media. Proteins were extracted from cells after $24 \mathrm{~h}$; protein extracts were then subjected to Western blotting using specific primary antibodies. Similar results were obtained in two different experiments.

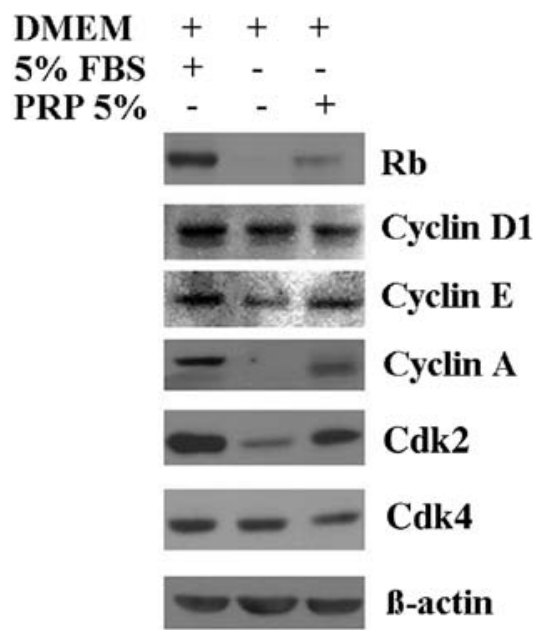

Figure 4. Expression of cell cycle regulatory proteins in PRP (high concentrations)-treated fibroblasts. Cells were cultured without serum for 3 days, followed by addition of 5\% FBS or 5\% PRP to culture media. Proteins were extracted from cells after $24 \mathrm{~h}$; protein extracts were then subjected to Western blotting using specific primary antibodies. Similar results were obtained in two different experiments.

high concentration (5\%) of PRP induced an increased expression of $\mathrm{Rb}$, cyclin $\mathrm{E}$, and $\mathrm{Cdk} 2$, as well as cyclin $\mathrm{A}$, in skin fibroblasts (Fig. 4).

Increased expression of type I collagen, MMP-1 and MMP-2 in skin fibroblasts by PRP treatment. To determine the effect of PRP on the expression of type I collagen, MMP-1 and MMP-2 in skin fibroblasts, fibroblasts were cultured by DMEM supplemented with 5\% PRP. First, we performed the CAT assay for evaluation of the promoter activity of type I collagen in PRP-treated fibroblasts. Promoter activity of type I collagen was markedly increased at 6 and 24 hafter PRP treatment in fibroblasts (Fig. 5). Expression of type I collagen protein was 


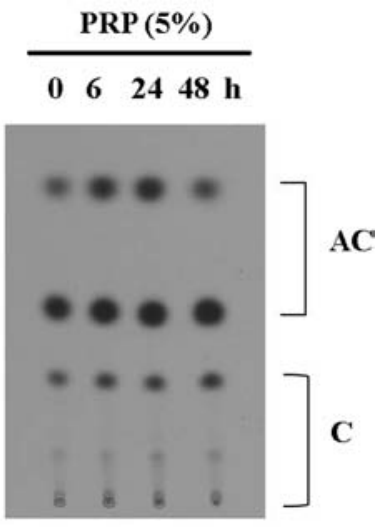

Figure 5. Increased activity of $\alpha 1$ (I) procollagen promoter in PRP-treated fibroblasts. Confluent fibroblasts were transfected with CAT constructs containing the proximal region of the human $\alpha 1$ (I) procollagen promoter. CAT activity was determined by autoradiography in the acetylated and unacetylated forms of $\left[{ }^{14} \mathrm{C}\right]$ chloramphenicol. Similar results were observed in two different experiments. AC, acetylated chrolamphenicol; $\mathrm{C}$, chloramphenicol.

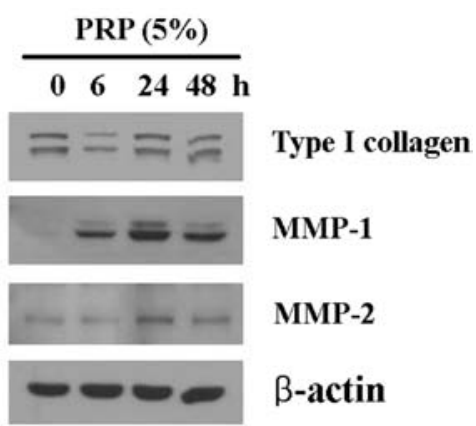

Figure 6. Increased type I collagen, MMP-1, and MMP-2 expression in PRPtreated fibroblasts. Cells were treated with PRP (5\%) for a period of $24 \mathrm{~h}$ Expression of type I collagen, MMP-1 and MMP-2 proteins was determined by Western blotting. Similar results were observed in two different experiments.

increased at 24 and $48 \mathrm{~h}$ after PRP treatment (Fig. 6A). In addition, PRP induced an increase in the expression of MMP-1 and MMP-2. Consistent with this data, increased MMP-2 protein activity was confirmed by zymography (Fig. 6B).

ERK-dependent MMP-1 expression in PRP-treated skin fibroblasts. We examined the effect of PRP on activation of p38 MAPK, JNK, and ERK in fibroblasts. Treatment with PRP resulted in phosphorylation (activation) of p38 and ERK (Fig. 7). Activation of p38 and ERK became apparent at 10 and 5 min following PRP treatment, respectively. Stripping and reprobing of the same membrane with antibodies against p38 MAPK and ERK revealed no change in total protein levels of each kinase, indicating that PRP-induced activation of pre-existing p38 MAPK and JNK. To confirm p38 MAPK-dependent or ERK-dependent MMP-1 up-regulation, fibroblasts were pre-treated with SB203580 $(25 \mu \mathrm{M})$ or PD98059 $(50 \mu \mathrm{M})$, and the cells were then treated with PRP. The $\mathrm{p} 38$ inhibitor, SB203580, and the ERK inhibitor, PD98059, specifically inhibited activation of $\mathrm{p} 38$ and JNK, respectively (data not shown). PRP-induced MMP-1 expression was attenuated by pre-treatment with PD98059, but not with the p38 inhibitor, SB203580 (Fig. 8).

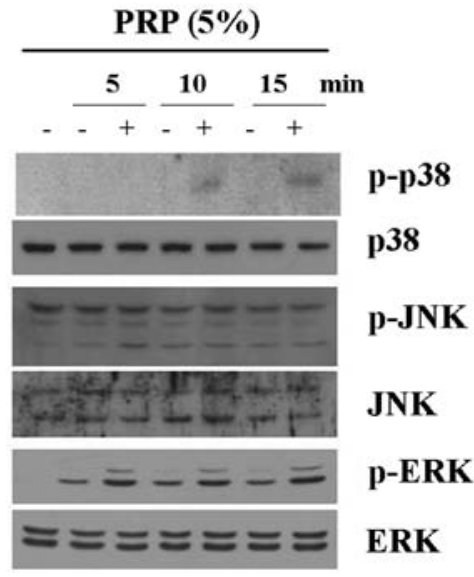

Figure 7. Increased activity of $\mathrm{p} 38$ and ERK proteins in PRP-treated fibroblasts. After PRP treatment, cells were harvested at the indicated times. At each time, whole cell lysates were prepared and used for p-p38, p38, p-JNK, JNK, p-ERK and ERK Western blotting with respective antibodies.

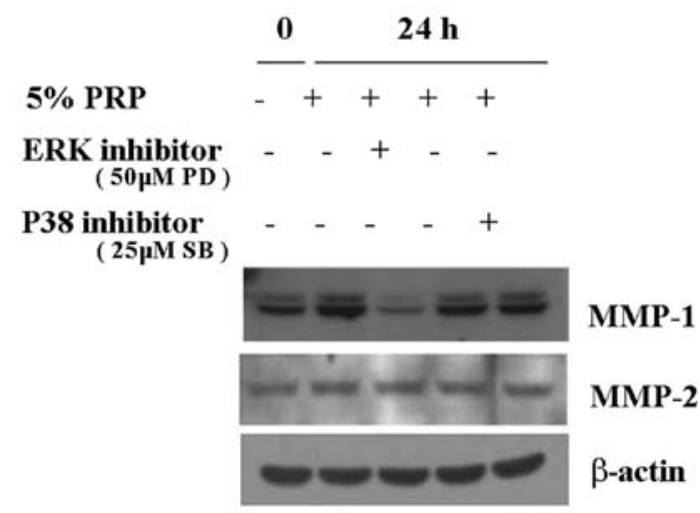

Figure 8. PRP-induced MMP-1 expression through the ERK pathway in fibroblasts. Cells were pre-treated with the p38 inhibitor, SB203580 $(25 \mu \mathrm{M})$ or the ERK inhibitor, PD98059 $(50 \mu \mathrm{M})$ for a period of $30 \mathrm{~min}$, followed by treatment with 5\% PRP. Whole cell lysates were prepared and used for MMP-1 or MMP-2 Western blotting with respective antibodies.

\section{Discussion}

Fibroblasts are the most common cell type in the dermis and the most influential cells in production of collagen and other ECM components; therefore, their role in the wound repair process has become fundamental to the understanding and evaluation of growth factor therapies in chronic wounds. Of particular interest, cultured fibroblasts from chronic skin ulcers showed characteristics of replicative senescence cells, implying a state of quiescence $(11,14)$. In addition, premature fibroblast senescence may contribute to delay of the wound healing process in venous leg ulcers and has been associated with poor prognosis $(15,16)$. Thus, in order to achieve completion of the wound healing process, some growth factors must be released from platelets or injected into wound areas at certain threshold concentrations.

Alpha granules within platelets contain more than 30 bioactive proteins, many of which have a fundamental role in hemostasis and wound healing $(17,18)$. These proteins include PDGF, TGF- $\beta$, VEGF, EGF and bFGF. Thus, autologous PRP therapy might be a promising agent for acceleration of the 
wound healing process through high concentrations of growth factors.

Fibroblasts are strongly reactive to bFGF, PDGF and EGF. In this study, we have attempted to investigate the effect of PRP on fibroblast activation, such as, migration, proliferation, and expression of G1 cell cycle regulatory proteins. Our data clearly demonstrate that PRP induced increases in cell migration, and proliferation rates, as well as an increase in the expression of $\mathrm{G} 1$ cell cycle regulatory proteins, such as cyclin $\mathrm{A}, \mathrm{Cdk} 2$, and cyclin $\mathrm{E}$ in HSF. Accumulating data indicate that growth factors and cytokines control cell proliferation through regulation of cell cycle progression (19). Growth factors promote entry into and progression through the cell cycle by regulating expression levels and activation of cyclin-Cdks. IGF-I induces an increase in mRNA levels of cyclin D1, E, A and B1, thereby promoting quiescent cells to enter G1 of the cell cycle (20). These data indicate that the PRP-induced increases in the expression of cyclin A, Cdk2, and cyclin E may play important roles in acceleration of the wound healing process through activation of fibroblasts.

MMPs promote remodeling of extracellular matrix, thus facilitating cell migration, and are implicated in the wound healing process. In this study, treatment of HSF with PRP resulted in increased expression of type I collagen, MMP-1, and MMP-2. PRP-induced MMP-1 up-regulation was mediated by an ERK-dependent signaling pathway. MMP-1 is able to cleave components of cell-cell junctions and cell-matrix contacts within the epithelium to promote re-epithelialization. MMP-1 is present in human cutaneous wounds during re-epithelialization, but switches off once wound closure is completed. In chronic wounds in which ECM has shown signs of development, it is possible that remaining growth factors become bound to the ECM and are unavailable for repair. Insulin-like growth factor can be bound by a binding protein to ECM (21). Likewise, granulocyte-macrophage colony-stimulating factor may be bound to the ECM and unavailable for participation in the wound repair process (22). Thus, the PRP-induced alterations in the expression of MMPs may trigger the wound healing process through release of ECM-bound growth factors.

In conclusion, our data suggest that PRP treatment plays an important role in promotion of wound healing in skin through promotion of proliferation and migration of fibroblasts, up-regulation of type I collagen, MMP-1 and MMP-2 expression in fibroblasts.

\section{Acknowledgements}

This study was supported by the research promoting grant from the Keimyung University Dongsan Medical Center in 2010 .

\section{References}

1. Arora NS, Ramanayake T, Ren YF and Romanos GE: Plateletrich plasma: a literature review. Implant Dent 18: 303-310, 2009.

2. Azzena B, Mazzoleni F, Abatangelo G, Zavan B and Vindigni V: Autologous platelet-rich plasma as an adipocyte in vivo delivery system: case report. Aesthetic Plast Surg 32: 155-161, 2008.

3. Bir SC, Esaki J, Marui A, et al: Angiogenic properties of sustained release platelet-rich plasma: characterization in vitro and in the ischemic hind limb of the mouse. J Vasc Surg 50: 870-879, 2009.

4. Carlson NE and Roach RB: Platelet-rich plasma: clinical applications in dentistry. J Am Dent Assoc 133: 1383-1386, 2002.

5. Barrientos S, Stojadinovic O, Golinko MS, Brem H and TomicCanic M: Growth factors and cytokines in wound healing. Wound Repair Regen 16: 585-601, 2008.

6. McDougall S, Dallon J, Sherratt J and Maini P: Fibroblast migration and collagen deposition during dermal wound healing: mathematical modelling and clinical implications. Philos Transact A Math Phys Eng Sci 364: 1385-1405, 2006.

7. Johnstone CC and Farley A: The physiological basics of wound healing. Nurs Stand 19: 59-65, 2005

8. Velnar T, Bailey T and Smrkolj V: The wound healing process: an overview of the cellular and molecular mechanisms. J Int Med Res 37: 1528-1542, 2009

9. Sottile J, Mann DM, Diemer V and Millis AJ: Regulation of collagenase and collagenase mRNA production in early- and late-passage human diploid fibroblasts. J Cell Physiol 138: 281-290, 1989.

10. Vande Berg JS and Robson MC: Arresting cell cycles and the effect on wound healing. Surg Clin North Am 83: 509-520, 2003.

11. Vande Berg JS, Rudolph R, Hollan C and Haywood-Reid PL: Fibroblast senescence in pressure ulcers. Wound Repair Regen 6: 38-49, 1998.

12. Neganova I and Lako M: G1 to S phase cell cycle transition in somatic and embryonic stem cells. J Anat 213: 30-44, 2008.

13. Donjerkovic D and Scott DW: Regulation of the G1 phase of the mammalian cell cycle. Cell Res 10: 1-16, 2000.

14. Harding KG, Moore K and Phillips TJ: Wound chronicity and fibroblast senescence - implications for treatment. Int Wound J 2: 364-368, 2005

15. Stanley A and Osler T: Senescence and the healing rates of venous ulcers. J Vasc Surg 33: 1206-1211, 2001.

16. Mendez MV, Stanley A, Park HY, Shon K, Phillips T and Menzoian JO: Fibroblasts cultured from venous ulcers display cellular characteristics of senescence. J Vasc Surg 28: 876-883, 1998.

17. Rendu F and Brohard-Bohn B: The platelet release reaction: granules' constituents, secretion and functions. Platelets 12: 261-273, 2001

18. Harrison P and Cramer EM: Platelet alpha-granules. Blood Rev 7: 52-62, 1993.

19. Stull MA, Rowzee AM, Loladze AV and Wood TL: Growth factor regulation of cell cycle progression in mammary epithelial cells. J Mammary Gland Biol Neoplasia 9: 15-26, 2004.

20. Stull MA, Richert MM, Loladze AV and Wood TL: Requirement for IGF-I in epidermal growth factor-mediated cell cycle progression of mammary epithelial cells. Endocrinology 143: 1872-1879, 2002.

21. Goldstein S, Moerman EJ and Baxter RC: Accumulation of insulinlike growth factor binding protein-3 in conditioned medium of human fibroblasts increases with chronologic age of donor and senescence in vitro. J Cell Physiol 156: 294-302, 1993.

22. Gordon MY, Riley GP, Watt SM and Greaves MF: Compartmentalization of a haematopoietic growth factor (GM-CSF) by glycosaminoglycans in the bone marrow microenvironment. Nature 326: 403-405, 1987. 